\title{
33 DEVELOPMENT OF A CLINICAL EX VIVO ASSAY FOR THE ASSESSMENT OF THERAPEUTIC CD28 COSTIMULATORY PATHWAY ENGAGEMENT
}

Chelsea Gudgeon*, Mark Maurer, Gary Means, Sherri Mudri, Lori Blanchfield, Jing Yang, Stacey Dillon, Pamela Holland, Zelanna Goldberg, Stanford Peng. Alpine Immune Sciences, Inc., Seattle, WA, USA

Background Preclinical evidence supports combining checkpoint inhibition (CPI) with $\mathrm{T}$ cell costimulatory agonism to improve the breadth and durability of anti-tumor responses relative to CPI alone. Currently, there are a number of therapeutic approaches combining costimulatory receptor agonists (e.g. CD28, 4-1BB, OX40L, etc.) with tumor targeting agents and/or CPI. Identification of a pharmacodynamically-justified therapeutic dose can be challenging because traditional duration of target occupancy does not necessarily correlate with immunological activity in the case of costimulatory molecules, and an 'always on' dose risks immune exhaustion. ALPN-202, a variant CD80 vIgD-Fc fusion protein that mediates PD-L1dependent CD28 costimulation and inhibits the PD-L1 and CTLA-4 checkpoints, is in development for the treatment of multiple advanced malignancies. To assess clinical CD28 agonism in the context of ALPN-202 treatment, we developed a novel, ex vivo whole blood target-dependent costimulation (TDC) assay.

Methods A TDC assay was developed using clinical samples from NEON-1 (NCT04186637), an ongoing dose escalation and expansion clinical trial of ALPN-202 for patients with advanced malignancies. The assay uses patient blood stimulated with paraformaldehyde-fixed, artificial antigen presenting cells (aAPC) expressing both cell-surface anti-CD3 and PD-L1. Pre-dose and end-of-infusion (EOI) blood was drawn from trial participants and co-cultured for 24 hours with the aAPCs in a pre-made assay plate. Plasma was collected and secreted IL-2 was quantified and used as a measure of PD-L1-dependent CD28 costimulation. Nonlinear regression was used to calculate area under the curve (AUC) for each condition, and sample AUC values were compared to a positive control (predose blood stimulated with a fixed concentration of ALPN202). Serum concentration of ALPN-202 and CD28 target saturation analyses were conducted concurrently to evaluate the exposure-response relationship.

Results Using the ex vivo TDC assay, ALPN-202 demonstrated PD-L1-dependent $\mathrm{T}$ cell costimulation at all dose levels tested to date in the NEON-1 clinical trial, consistent with preclinical assay development data. Similarly, CD28 target saturation levels on circulating $\mathrm{T}$ cells correlated with serum concentration of ALPN-202.

Conclusions We have developed a novel ex vivo assay to assess induction of PD-L1-dependent CD28 costimulation in a therapeutic setting. This assay has been successfully employed to monitor controlled CD28 costimulation by the CD28 agonist therapeutic candidate ALPN-202, helping to establish a $\mathrm{PK} / \mathrm{PD}$ relationship that is consistent with preclinical data. More broadly, this type of cell-based, ex vivo TDC assay could be adapted to assess costimulatory receptor engagement, particularly target-dependent costimulation, for other therapeutic agonists in clinical development.

Ethics Approval This study was approved by WCG IRB's Human Subjects Review, approval number: 20211877.

http://dx.doi.org/10.1136/jitc-2021-SITC2021.033 